\title{
Fabrication of Nanocables by Electrochemical Deposition inside Metal Nanotubes
}

Jie-Ren Ku, Ruxandra Vidu, Raisa Talroze and Pieter Stroeve*

Department of Chemical Engineering and Materials Science, University of California, Davis,

One Shields Avenue, Davis, California 95616.

\section{Supporting Information}

Nanocables offer unusual opportunities to explore properties at nanoscale dimensions and demonstrate a wide range of potentially useful applications. We have created single nanocables with uniform layers. The technology of creating metal/semiconductors and metal/semiconductors/metal nanocables that are co-axially grown, lays down a platform for constructing a group of previously unavailable materials. We have demonstrated the new technique with $\mathrm{Au} / \mathrm{Te}$ nanocables of about $100 \mathrm{~nm}$ in diameter and a several micrometers in length. The final product is a nano-sized coaxial cable, with a Te core and an Au sheath. The layer composition of the nanocables can be tailored for a wide variety of applications. Nanocables of may be used as nanodevices such as biosensors, nanoelectronics, solar cells, and nano-electrical-mechanical devices. Fabrication of an array of nanocables is also possible.

\section{Background}

This part briefly presents the use of nanoporous membranes in combination with electrochemical deposition or electrodeposition for creating nanocables containing the semiconductor cores and metal shell. The process is shown in Fig. 1.

Step 1. Using a nanoporous membrane (Poly-carbonate track-etching membrane, anodic aluminum oxide membrane, Si membrane, and etc.) as the first template (Fig. 1a), metal (Au, $\mathrm{Ag}$, and etc.) nanotubular membranes were obtained by electroless deposition of metal (Fig. 1b).

Step 2 Using the metal nanotubular membranes as the secondary templates (working electrode), semiconductor material ( $\mathrm{Te}$, and $\mathrm{CdTe}, \mathrm{CdS}, \mathrm{ZnS}$, and etc.) are grown inside of the metal nanotubes by electrochemical deposition at a fixed potential, either as nanowires or as nanotubes. The potential chosen is between bulk deposition and UPD (onset underpotential deposition) so that the growth is at a low deposition rate. In this manner, the deposition of semiconductor material forms nanowires or nanotubes inside the metal nanotubes. Multilayer nanocables are obtained by repeating the electrochemical deposition steps for different materials.

Step 3 Removal of the first template and eventualy the Au nanotube can be achieved by chemical etching methods. Nanocables in array format are obtained by electrochemical deposition of semiconductors ( $\mathrm{Te}$, and $\mathrm{CdTe}, \mathrm{CdS}, \mathrm{ZnS}$, and etc.) outside or both-side of the metal nanotubes as described in Step 2a. The nanocable with multilayers is obtained by repeating the electrochemical deposition steps (Fig. 1c).

(a)

\section{Nanoporous membrane as the first template}

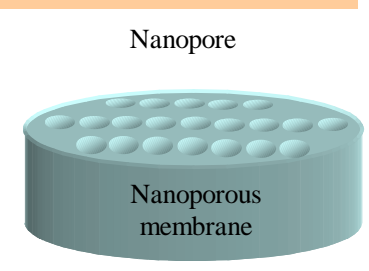

(b)
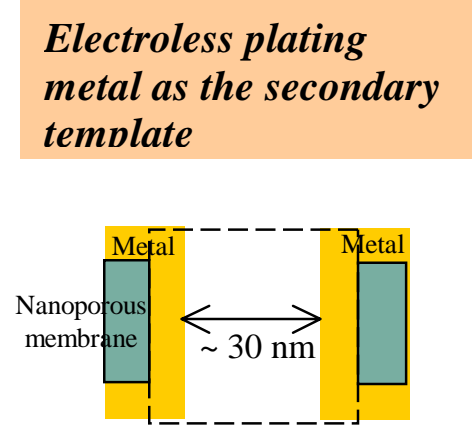

(c)

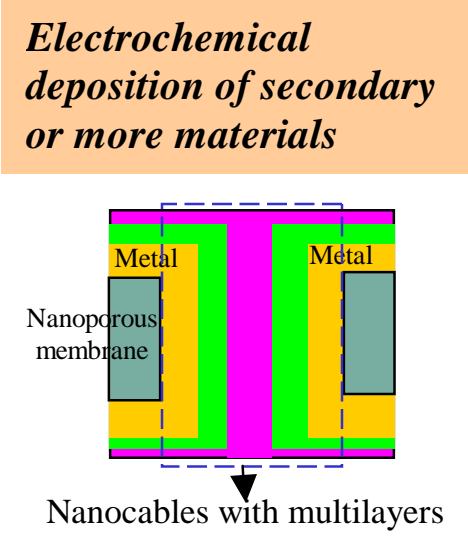

Figure 1. (a) Bare PCTE membrane. (b) Metal nanotubular membrane. (c) Secondary materials inside metal nanotubes. 


\section{$\underline{\text { Results }}$}

Nanocables, of $\mathrm{Au} / \mathrm{Te}$ composition, were characterized using various characterization techniques. The techniques used include: Energy Dispersive X-ray (EDX) spectroscopy, in situ Electrochemical Atomic Force Microscopy (EC-AFM), Scanning Electron Microscopy (SEM) and Transmission Electron Microscopy (TEM), Surface Plasmon Resonance (SPR) and Electron Energy Loss Spectrum Detector (EELS), which operated on a scanning transmission electron microscope (STEM). Here we present only a few additional results. A full article is in preparation. Figure $2 \mathrm{a}$ presents a SEM image of Au-Te nanocables glued to copper tape. The tape was glued to the membrane after Te deposition and then the polycarbonate was chemically etched away. The TEM image (Fig. 2b) shows a few Au-Te nanocables after their treatment with $\mathrm{Au}$ etchant. Figure 3 shows the EDX spectra for $\mathrm{Au}-\mathrm{Te}$ nanocable. In a separate experiment (in preparation) we made Te nanotubules from and Fig. 4 shows a SEM image of a single Te nanotube after complete Au dissolution, together with the EELS chemical profile across the nanotube along the indicated line 1 shown in (a). These results confirm the existence of Te in $\mathrm{Au} / \mathrm{Te}$ nanocable and nanotube structures, before and after Au dissolution respectively.
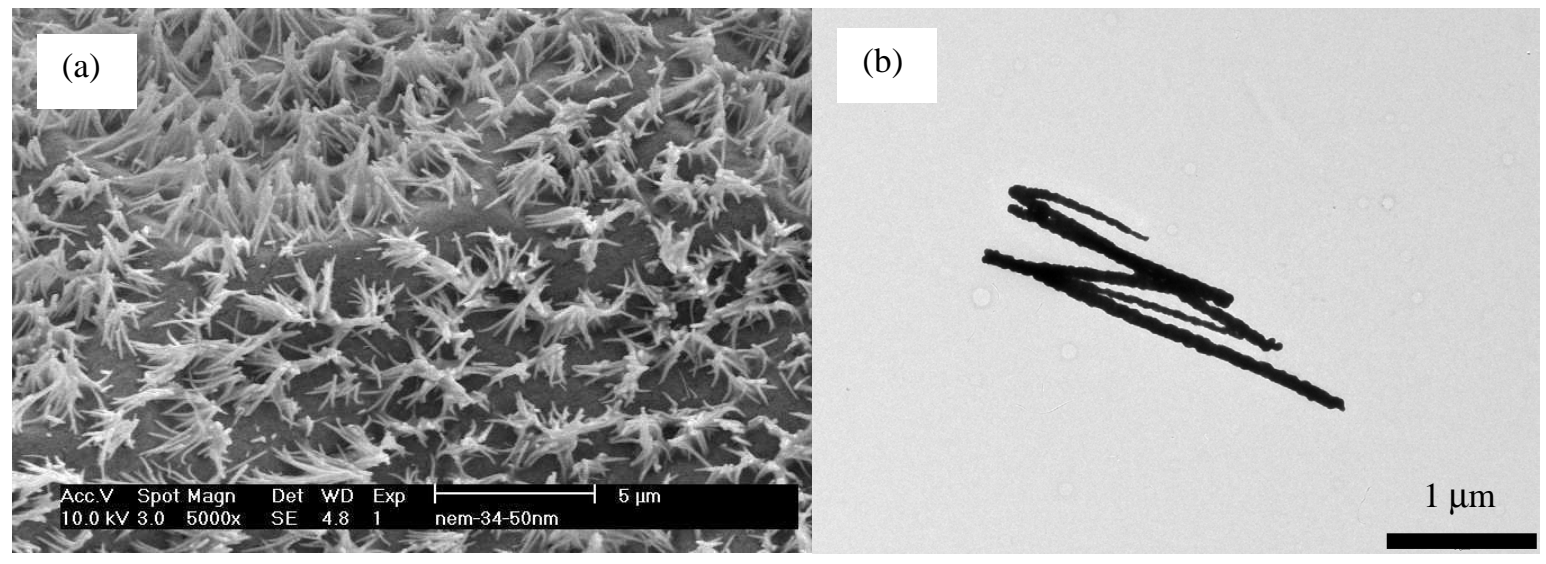

Figure 2. SEM and TEM images of Au-Te nanocables: (a) SEM image shows large amount of Au-Te nanocables; (b) TEM image shows a few Au-Te nanocables after treatment with $\mathrm{Au}$ etchant to remove $\mathrm{Au}$.

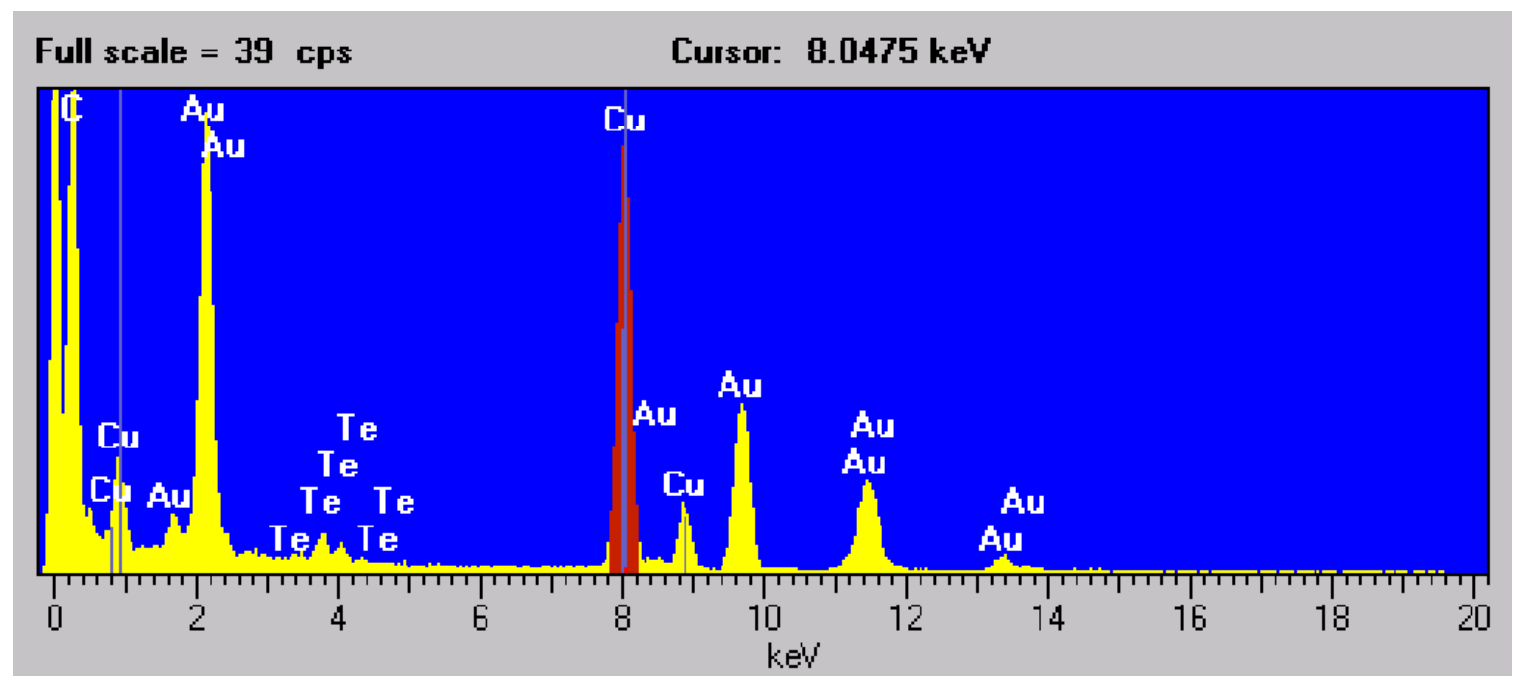

Figure 3 EDX spectra for Te nanocable 


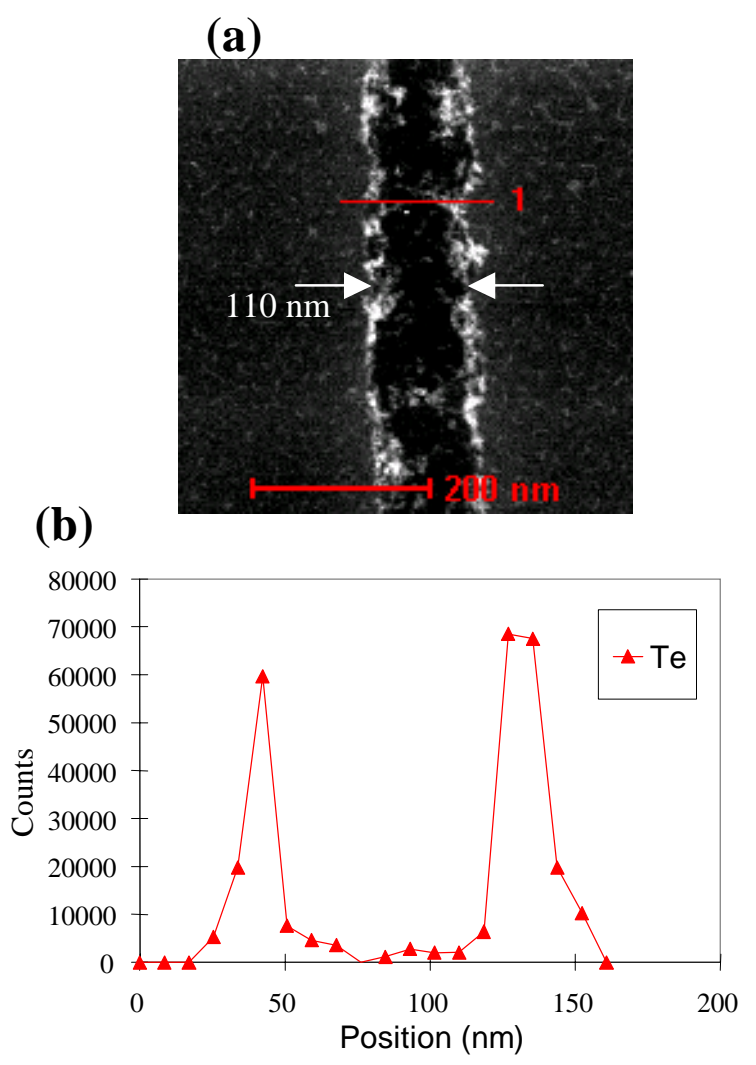

Figure 4. SEM image (a) of single Te nanotube after complete Au dissolution from Au/Te nanotube; (b) EELS chemical profile across the Te nanotube along the indicated line 1 shown in (a).

\section{$\underline{\text { Applications }}$}

Transistors: Cylindrically shaped transistors, nanoplugs or short nanocables, can be fabricated into an array of nanowells in silicon. Nanowells are shallow pores or dead-end pores and these can be fabricated into the silicon surface as a regular array. The shallow pores can have diameters as small as $20 \mathrm{~nm}$. Our method can be used to fabricate cylindrical transistors inside the nano wells. Thus transistors can now be made into the silicon itself. The short nanocables can have metal/semiconductor $1 /$ semiconductor $2 /$ semiconductor $3 /$ metal geometry.

Biosensors: The semiconductor-metal junction in a nanocables provides distinct voltages that shift due to the charges attached to the nanocable's surface when it comes into contact with molecules. Moreover, the signal voltage modulates the surface charge density. The use of nanocables as biosensors has several advantages over nanotubes:

1. While other nanostructures (e.g. carbon nanotubes) sense only the presence of molecules (as long as the molecule can bring the charges onto the nanotube surface), nanocables can distinguish among molecules due to the voltage measured at the junction.

2. The junction barrier is tunable in nanocables by doping the semiconductor layer or by applying external potential and it can be "custom" designed to detect specific molecules. 
3. Nanocables in array format have higher sensitivity than a single carbon nanotube, and permit the analysis of very low-concentration systems.

4. Nanocables in array format can sense more types of molecules in a toxic mixture compared to single carbon nanotube.

Solar Cells: For traditional thin film photovoltaic devices, a large surface area is always desirable to increase power. Solar cells based on semiconductor/metal nanocables (e.g. CdTe/Au nanocables) enhance the efficiency. The metal collector electrode can be very thin c.a. $20 \mathrm{~nm}$, which will not interfere with incoming light. The nanocable array provides a large surface area, which is 1000 times larger than that of a planar substrate for the same device size. Therefore, the power collected in a unit area is 1000 higher than that in the traditional photodiode solar cell.
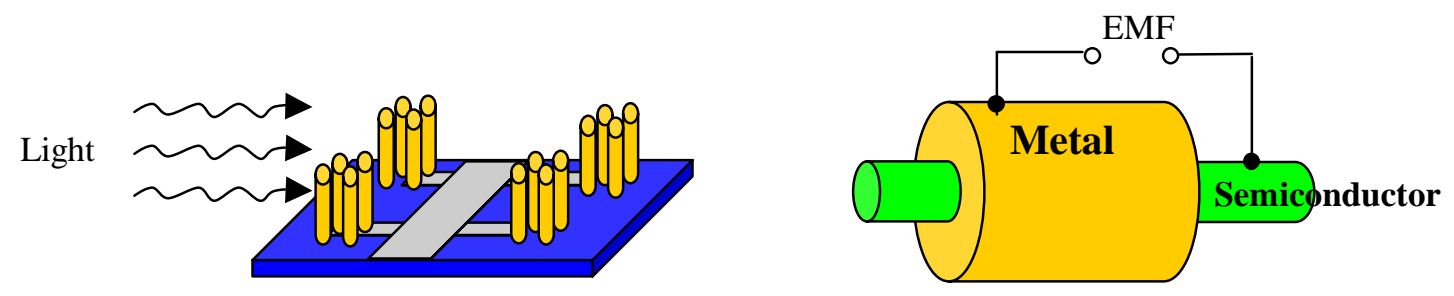

Figure 5. Nanocable solar cell: large surface area in array format (left), single nanocable (right).

Nanodevices: Semiconductor-metal junctions are considered nanodevices, such as diodes, transistors, and logical gates. According to our technology, nanodevices are easily integrated in a Si-packed wafer with electronic circuits.

NEMS (Nano-Electrical-Mechanical System) Nano-actuators, which work as piezo-electrics, can be obtained having a nanocable structure of metal $/ 2^{\text {nd }}$-material. 